EPJ Web of Conferences 21, 01001 (2012)

DOI: $10.1051 /$ epjconf/20122101001

(C) Owned by the authors, published by EDP Sciences, 2012

\title{
Neutron-capture cross sections from indirect measurements
}

\author{
J.E. Escher, ${ }^{\text {a }, ~ J . T . ~ B u r k e, ~ F . S . ~ D i e t r i c h, ~ J . J . ~ R e s s l e r, ~ N . D . ~ S c i e l z o, ~ a n d ~ I . J . ~ T h o m p s o n ~}$ \\ Lawrence Livermore National Laboratory, Livermore, CA 94550, USA
}

\begin{abstract}
Cross sections for compound-nuclear reactions reactions play an important role in models of astrophysical environments and simulations of the nuclear fuel cycle. Providing reliable cross section data remains a formidable task, and direct measurements have to be complemented by theoretical predictions and indirect methods. The surrogate nuclear reactions method provides an indirect approach for determining cross sections for reactions on unstable isotopes, which are difficult or impossible to measure otherwise. Current implementations of the method provide useful cross sections for $(\mathrm{n}, \mathrm{f})$ reactions, but need to be improved upon for applications to capture reactions.
\end{abstract}

\section{Introduction}

Nuclear reaction data play an important role in nuclear physics applications. Cross sections for reactions of neutrons and light, charged particles with target nuclei across the isotopic chart, taking place at energies from several $\mathrm{keV}$ to tens of $\mathrm{MeV}$, are required for nuclear astrophysics, national security, and nuclear energy applications. Not all relevant data can be directly measured in the laboratory as many important reactions involve unstable nuclei which are too difficult to produce with currently available techniques. Cross section calculations are nontrivial since they often require a thorough understanding of both direct and statistical reaction mechanisms (as well as their interplay) and a detailed knowledge of nuclear structure.

Several indirect methods are presently used to determine cross sections that are difficult to measure directly. The Asymptotic Normalization Coefficient (ANC), Coulomb-Dissociation, and Trojan-Horse methods aim at obtaining direct reaction cross sections ${ }^{1}$, while the surrogate nuclear reaction method is an indirect approach for determining compound-nuclear reaction cross sections. The method was first explored in the 1970s, when it was applied to estimate (n,f) cross sections. In the past several years, the approach has received renewed attention. Most of the applications so far have focused on obtaining cross sections for neutron-induced fission, but more recently attention has also turned to the question whether neutron capture cross sections can be obtained using this method.

The idea of applying the surrogate approach to neutron capture is certainly compelling, as capture cross sections play an important role in many applications, such as nuclear energy and nuclear astrophysics. Simulations of the evolution of stars and the synthesis of the heavy elements require capture cross sections as input. Many reactions involve unstable nuclei that cannot presently be measured in the laboratory. Even close to stability there are important reactions that are difficult or impossible to access directly. This includes selected neutron capture reactions that are relevant to the slow neutron capture process (s process) that, together with the rapid neutron capture process ( $\mathrm{r}$ process) produces almost all the elements heavier than iron. The s process involves nuclei in and very near the valley of stability. Of particular interest are capture reactions on s process branch points, unstable nuclei with a life time long enough to allow the s process to proceed by either neutron capture or $\beta$ decay. The strength with which one path dominates over the other depends on environmental variables, such as neutron density, temperature, and pressure, as well as on nuclear properties, specifically capture rates

\footnotetext{
a e-mail: escher1@llnl.gov

1 The Trojan-Horse method has recently also been explored for resonance reactions [1]
}

This is an Open Access article distributed under the terms of the Creative Commons Attribution-Noncommercial License 3.0, which permits unrestricted use, distribution, and reproduction in any noncommercial medium, provided the original work is properly cited 


\section{EPJ Web of Conferences}

and beta-decay life times. Information on the astrophysical conditions of the s process can be inferred if the nuclear properties are known. The desired accuracy for s-process cross sections used by stellar modelers lies in the single-digit percent range and is difficult to obtain, in particular for an indirect approach. However, in the absence of data, one finds that calculated cross sections exhibit large uncertainties. For instance, for s-process branch points ${ }^{95} \mathrm{Zr}$ and ${ }^{153} \mathrm{Gd}$ one finds that the evaluated (n, $\gamma$ ) cross sections in the literature differ by a factor of two or more.

The reason for the large differences between the evaluated cross sections can be found in the nuclear structure input that is required for Hauser-Feshbach calculations. The expression for the cross sections involves, among others, transmission coefficients and level densities. To calculate the former, one needs optical potentials, $\gamma$-ray strength functions, and - when fission competes - fission barriers. For level densities, there exist several analytic functions, with parameters adjusted to available data, as well as data tables based on microscopic approaches. Much work has been done to develop models, codes and parameter recommendations (see, e.g. [2]) for use in Hauser-Feshbach calculations. However, a given set of cross section data can often be reproduced by several parameter combinations. This ambiguity reflects our insufficient knowledge of the underlying nuclear structure properties at the energies relevant to compound-nuclear reactions. Consequently, for cases where there is little or no data to constrain the structure models and cross sections, we find discrepant cross section predictions. The question we want to consider here is whether the surrogate method can possibly be used to provide meaningful constraints to the inputs for the calculations.

\section{The surrogate idea}

The appropriate formalism for the description of a compound-nuclear reaction $a+A \rightarrow B^{*} \rightarrow c+C$ is a statistical one [3]. Based on Bohr's hypothesis of the independence of formation and decay of the compound nucleus $(\mathrm{CN})$, the cross sections are calculated in the framework of the Hauser-Feshbach formalism, which properly takes account of the conservation of angular momentum and parity in the reaction:

$$
\sigma_{\alpha \chi}\left(E_{a}\right)=\sum_{J, \pi} \sigma_{\alpha}^{C N}\left(E_{e x}, J, \pi\right) G_{\chi}^{C N}\left(E_{e x}, J, \pi\right)
$$

with $\alpha$ and $\chi$ denoting the relevant entrance and exit channels, $a+A$ and $c+C$, respectively. The excitation energy $E_{e x}$ of the compound nucleus, $B^{*}$, is related to the center-of-mass energy $E_{a}$ in the entrance channel via the energy needed for separating $a$ from $B: E_{a}=E-S_{a}(B)$. In many cases the formation cross section $\sigma_{\alpha}^{C N}=\sigma\left(a+A \rightarrow B^{*}\right)$ can be calculated to a reasonable accuracy by using optical potentials, while the theoretical decay probabilities $G_{\chi}^{C N}$ for the different decay channels $\chi$ are often quite uncertain. The latter are difficult to calculate accurately since they require knowledge of optical models, level densities, and strength functions for the various possible exit channels. The objective of the surrogate method is to determine or constrain these decay probabilities experimentally.

In the surrogate approach, the compound nucleus $B^{*}$ is produced by means of an alternative ("surrogate"), direct reaction, $d+D \rightarrow b+B^{*}$, and the desired decay channel $\chi\left(B^{*} \rightarrow c+C\right)$ is observed in coincidence with the outgoing particle $b$ (see Fig. 1). The probability for forming $B^{*}$ in the surrogate reaction (with specific values for $\left.E_{e x}, J, \pi\right)$ is $F_{\delta}^{C N}\left(E_{e x}, J, \pi, \theta_{b}\right)$, where $\delta$ refers to the entrance channel reaction $D(d, b)$ and $\theta_{b}$ is the angle of the outgoing direct-reaction particle $b$ relative to the beam axis. The quantity

$$
P_{\delta \chi, \theta_{b}}\left(E_{e x}, \theta_{b}\right)=\sum_{J, \pi} F_{\delta}^{C N}\left(E_{e x}, J, \pi, \theta_{b}\right) G_{\chi}^{C N}\left(E_{e x}, J, \pi\right),
$$

which gives the probability that the compound nucleus $B^{*}$ was formed with energy $E_{e x}$ and decayed into channel $\chi$, can be obtained experimentally, by measuring $N_{\delta}$, the total number of surrogate events, and $N_{\delta \chi}$, the number of coincidences between the direct-reaction particle and the observable that identifies the relevant exit channel: $P_{\delta \chi}^{e x p}\left(E_{e x}, \theta_{b}\right)=N_{\delta \chi}\left(E_{e x}, \theta_{b}\right) / N_{\delta}\left(E_{e x}, \theta_{b}\right) \epsilon_{\delta}\left(E_{e x}\right)$. Here, $\epsilon_{\delta}\left(E_{e x}\right)$ denotes the efficiency for detecting the exit-channel $\chi$ (when in coincidence with the outgoing direct-reaction particle $b$ ). 

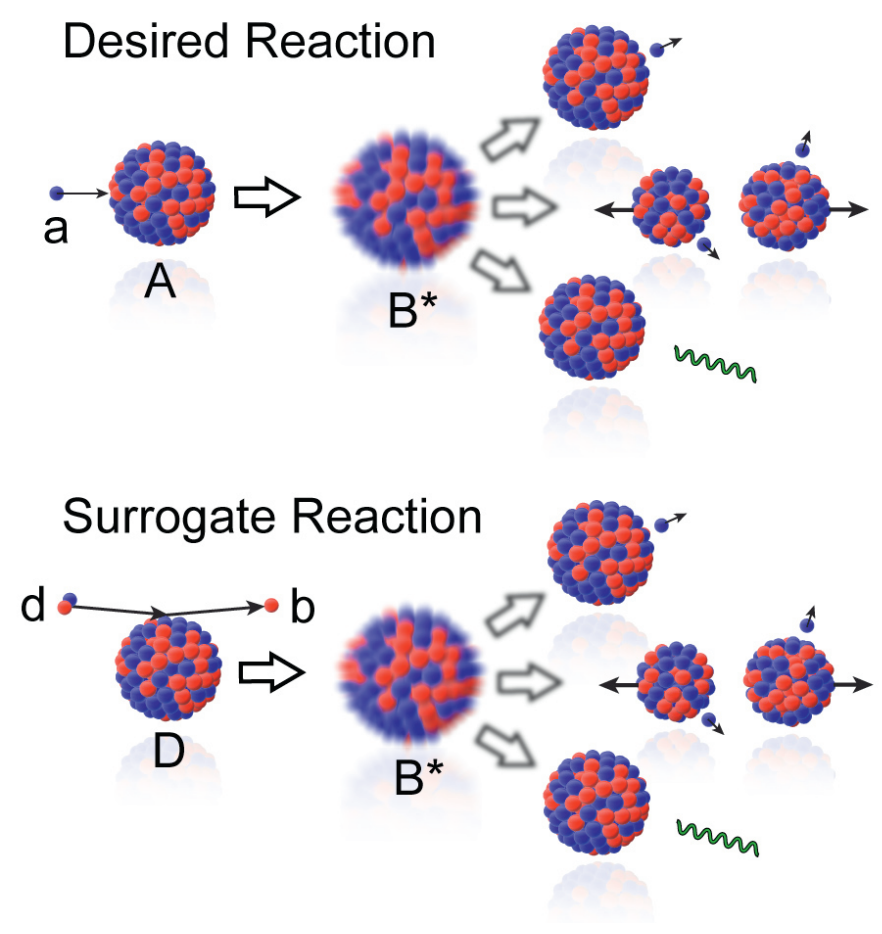

Fig. 1. Schematic representation of the "desired" (top) and "surrogate" (bottom) reaction mechanisms [4]. The basic idea of the surrogate approach is to replace the first step of the desired reaction, $a+A$, by an alternative (surrogate) reaction, $d+D \rightarrow b+B^{*}$, that is experimentally easier to access yet populates the same compound nucleus. The subsequent decay of the compound nucleus into the relevant channel, $c+C$, can then be measured and used to extract the desired cross section. Three typical decay channels are shown here: neutron evaporation, fission, and $\gamma$ emission.

The distribution $F_{\delta}^{C N}\left(E_{e x}, J, \pi, \theta_{b}\right)$, which may be very different from the $\mathrm{CN}$ spin-parity populations following the absorption of the projectile $a$ in the desired reaction, has to be determined theoretically, so that the branching ratios $G_{\chi}^{C N}\left(E_{e x}, J, \pi\right)$ can be extracted from the measurements. In practice, the decay of the $\mathrm{CN}$ is modeled and the $G_{\chi}^{C N}\left(E_{e x}, J, \pi\right)$ are obtained by adjusting parameters in the model to reproduce the measured probabilities $P_{\delta \chi}\left(E_{e x}, \theta_{b}\right)[5,6]$. Subsequently, the sought-after cross section can be obtained by combining the calculated cross section $\sigma_{\alpha}^{C N}\left(E_{e x}, J, \pi\right)$ for the formation of $B^{*}$ (from $a+A$ ) with the extracted decay probabilities $G_{\chi}^{C N}\left(E_{e x}, J, \pi\right)$ for this state, see Eq. 1. To simplify the notation, we suppress, in the remainder of the paper, the dependence of the coincidence probability $P_{\delta x}^{\exp }\left(E_{e x}, \theta_{b}\right)$ on the angle $\theta_{b}$ of the outgoing direct-reaction particle $b$. The angular dependence of $P_{\delta \chi}^{e x p}\left(E_{e x}, \theta_{b}\right)$ arises from the fact that the population $F_{\delta}^{C N}\left(E_{e x}, J, \pi, \theta_{b}\right)$ of the compound nucleus depends on the angular-momentum transferred in the surrogate reaction, and hence on $\theta_{b}$.

Weisskopf-Ewing Approximation. Under certain circumstances, the decay of the intermediate equilibrated system, the compound nucleus, becomes independent of its angular momentum and parity, and the cross section for the reaction factorizes into a simple product of a formation cross section and a decay probability for the exit channel of interest [3]:

$$
\sigma_{\alpha \chi}\left(E_{a}\right)=\sigma_{\alpha}^{C N}\left(E_{e x}\right) G_{\chi}^{C N}\left(E_{e x},\right)
$$

where $\sigma_{\alpha}^{C N}\left(E_{e x}\right)=\sum_{J, \pi} \sigma_{\alpha}^{C N}\left(E_{e x}, J, \pi\right)$ is the reaction cross section describing the formation of the compound nucleus in the desired reaction and $\mathcal{G}_{\chi}^{C N}\left(E_{e x}\right)$ denotes the $J^{\pi}$-independent decay probability for the exit channel $\chi$. This is the Weisskopf-Ewing (WE) limit of the Hauser-Feshbach theory [3]. 


\section{EPJ Web of Conferences}

In the context of surrogate reactions, the Weisskopf-Ewing approximation greatly simplifies the application of the method: It becomes straightforward to obtain the $J^{\pi}$-independent branching ratios $\mathcal{G}_{\chi}^{C N}\left(E_{e x}\right)$ from measurements of $P_{\delta \chi}\left(E_{e x}\right)\left[=\mathcal{G}_{\chi}^{C N}\left(E_{e x}\right)\right.$ since $\left.\sum_{J, \pi} F_{\delta}^{C N}\left(E_{e x}, J, \pi\right)=1\right]$ and to calculate the desired reaction cross section. Calculating the direct-reaction probabilities $F_{\delta}^{C N}\left(E_{e x}, J, \pi\right)$ and modeling the decay of the compound nucleus are no longer required. Most applications to date invoke the Weisskopf-Ewing approximation.

\section{Neutron-induced fission reactions}

The surrogate approach was first employed in the 1970s to estimate neutron-induced fission cross sections from transfer reactions. These early applications of the method made use of the WeisskopfEwing approximation: The transfer reactions of the 1970s [7,8] produced $(\mathrm{n}, \mathrm{f})$ cross section estimates for various actinide targets which agreed with direct measurements (where available) to about 10-20\% for incident neutron energies above $1 \mathrm{MeV}$. Discrepancies at lower energies were later attributed to large uncertainties in the low-energy optical model employed, and the use of the Weisskopf-Ewing approximation $[5,6]$.

More recently, a French group has carried out surrogate experiments at the Institut de Physique Nucléaire (IPN) in Orsay to determine cross sections for neutron-induced reactions on several minor actinide nuclei relevant to the thorium-uranium fuel cycle and the transmutation of nuclear waste [911]. The transfer reactions ${ }^{232} \mathrm{Th}\left({ }^{3} \mathrm{He}, \mathrm{x}\right)$ and ${ }^{243} \mathrm{Am}\left({ }^{3} \mathrm{He}, \mathrm{x}\right)$, with $\mathrm{x}=\alpha, \mathrm{t}, \mathrm{d}, \mathrm{p}$, were employed to obtain $(\mathrm{n}, \mathrm{f})$ and $(\mathrm{n}, \gamma)$ cross sections for Th and Pa targets $[9,10]$ and $(\mathrm{n}, \mathrm{f})$ cross sections for $\mathrm{Cm}$ and Am targets [11], respectively. The analyses assumed that the Weisskopf-Ewing approximation is valid. The extracted $(\mathrm{n}, \mathrm{f})$ cross sections were found to be consistent with known directly-measured cross section measurements, where these were available. They proved useful for resolving controversies between discrepant measurements and for providing data for previously unavailable energy regions.

The STARS/LiBerACE collaboration in the United States has carried out a number of experiments at the 88-inch cyclotron at Lawrence Berkeley National Laboratory. Light-ion beams have been used for inelastic scattering, charge exchange, and one- or two-neutron transfer reactions. Fission cross sections, e.g. the ${ }^{237} \mathrm{~Np}(\mathrm{n}, \mathrm{f})$ cross section [12], have been determined using the Weisskopf-Ewing approximation or a variant thereof, the surrogate ratio method.

The Surrogate Ratio approach [13-15] requires the (approximate) validity of the Weisskopf-Ewing limit. The ratio

$$
R(E)=\frac{\sigma_{\alpha_{1} \chi_{1}}}{\sigma_{\alpha_{2} \chi_{2}}}
$$

of the cross sections of two $\mathrm{CN}$ reactions is measured, using two surrogate experiments. An independent determination of the cross section $\sigma_{\alpha_{2} \chi_{2}}$ can then be used to deduce $\sigma_{\alpha_{1} \chi_{1}}$. An advantage of using the ratio method is the fact that it eliminates the need to accurately measure the total number of surrogate reaction events $\left(N_{\delta}\right)$, since one determines the ratio of coincidence probabilities $P_{\delta_{1} \chi_{1}} / P_{\delta_{2} \chi_{2}}$, rather than an absolute probability $P_{\delta \chi}=N_{\delta \chi} / N_{\delta}$. Furthermore, there are indications that small to moderate deviations from the WE limit cancel in this approach [13]. Cross sections for $(\mathrm{n}, \mathrm{f})$ reactions extracted in the ratio approximation have been tested for consistency with results from direct measurements, complementary surrogate experiments $[15,16]$, and theoretical simulations [13]. For (n,f) reactions one typically finds that the spin-parity mismatch between the desired and surrogate reactions has a much smaller effect on the extracted cross section than in an approach that uses the WE approximation and absolute probabilities. Also, deviations caused by pre-equilibrium effects are diminished, thus improving the overall agreement between extracted and expected cross sections.

A recent applications of the ratio approach is shown in Figure 3. The ${ }^{238} \mathrm{Pu}(\mathrm{n}, \mathrm{f})$ cross section, which is needed for reactor applications and for transmutation studies, was recently determined using inelastic scattering surrogate reactions [17]. Ressler et al. [17] produced the compound nuclei ${ }^{239} \mathrm{Pu}^{*}$, ${ }^{235} \mathrm{U}^{*}$, and ${ }^{236} \mathrm{U}^{*}$ via inelastic $\alpha$ scattering. Surrogate ratio analyses yielded the desired ${ }^{238} \mathrm{Pu}(\mathrm{n}, \mathrm{f})$ cross section relative to both the ${ }^{234} \mathrm{U}(\mathrm{n}, \mathrm{f})$ and the ${ }^{235} \mathrm{U}(\mathrm{n}, \mathrm{f})$ cross sections. Since the latter two are known, the ${ }^{238} \mathrm{Pu}(\mathrm{n}, \mathrm{f})$ cross section could be extracted; the weighted average of both measurements is shown 


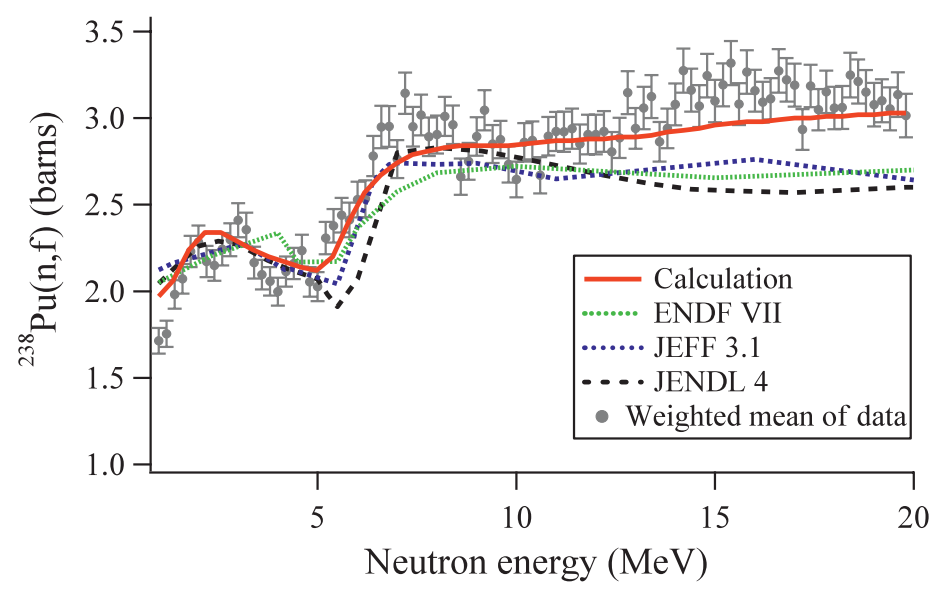

Fig. 2. Fission cross sections obtained from surrogate ratio measurements. The ${ }^{238} \mathrm{Pu}(\mathrm{n}, \mathrm{f})$ cross section was determined relative to the known ${ }^{234} \mathrm{U}(\mathrm{n}, \mathrm{f})$ and ${ }^{235} \mathrm{U}(\mathrm{n}, \mathrm{f})$ cross sections [17]. The weighted average of the two measurements is compared to several evaluations; the solid line represents a calculation based on the new data.

in the left panel of Figure 3. The two measurements are in good agreement with each other for 5-20 $\mathrm{MeV}$ and the averaged cross section agrees well with previous, direct, measurements in the 5-10 MeV range; it is somewhat higher (by less than 20\%) than those at about $15 \mathrm{MeV}$. The ratio measurement does not, and is not expected to, produce highly-accurate fission results at low energies $(<5 \mathrm{MeV}$ here), due to the underlying Weisskopf-Ewing assumption. It does, however, provide continuous data for neutron energies from 5 to $20 \mathrm{MeV}$, and supplement earlier measurements, which were sparse in the energy regime around 10-15 MeV.

The literature and the above examples indicate that $(n, f)$ cross sections extracted from surrogate data are typically consistent with direct measurements (where available) and/or other surrogate measurements, despite the approximations used. In addition, calculations, which test the approximation schemes employed in the analyses of surrogate fission data [13], illustrate the level of accuracy that one can under reasonable circumstances expect from the surrogate approach. Discrepancies between indirectly and directly measured cross sections are often less than $10 \%$. The largest deviations are found at low energies, where the Weisskopf-Ewing approximation is not expected to be valid; in those cases, it becomes necessary to account for the differences in the spin-parity distributions occurring in the desired and surrogate reactions.

\section{Capture reactions}

Capture cross sections provide specific challenges for the surrogate approach. First, the level of precision required for the cross section is often higher than in the fission case: Recent advances in modeling the astrophysical s process have resulted in requests to determine capture cross sections within a few percent and nuclear-energy applications require cross sections to within 5-10\% [18,19]. Achieving an accuracy of a few percent is challenging, but constraining an unknown (n, $\gamma$ ) cross section to within 20-30\% should be considered a meaningful improvement of the situation, in particular since current cross section evaluations often show large deviations from each other. Secondly, it is the low-energy regime that is relevant to many applications. For s-process applications, for example, one needs cross sections from a few $\mathrm{keV}$ to about $200 \mathrm{keV}$. Both calculations and measurements have shown that this is the energy range for which the Weisskopf-Ewing approximation typically breaks down.

Theoretical studies have been carried out to assess the feasibility of obtaining capture cross sections from surrogate measurements and to determine promising candidates for such measurements. The strategy followed in these investigations is to extract information from Hauser-Feshbach calculations that have been adjusted to reproduce known cross sections (capture and, where applicable, fission). 
The branching ratios (channel probabilities), $G_{\chi}^{C N}(E, J, \pi)$ that enter Eqs. 1 and 2 can be calculated via this procedure; the Weisskopf-Ewing limit is reached when the branching ratios are approximately equal.

Overall, the studies show that the probability for a compound nucleus to decay via $\gamma$ emission $(\chi=\gamma)$ depends sensitively on the spin-parity population of the nucleus prior to decay (see Fig. 3). The dependence of the $\gamma$-branching ratios on the $J \pi$ distribution is greater than that found previously for fission $(\chi=$ fission $)$. Calculations for representative Zirconium, Gadolinium, and Uranium nuclei showed a strong dependence of the $\gamma$ branching ratios on the spins populated in the compound nucleus. The effect was particularly strong for the ${ }^{92} \mathrm{Zr}$ nucleus, which has a closed proton subshell $(\mathrm{Z}=40)$ and a nearly-closed neutron shell $(\mathrm{N}=52 \approx 50)$ [20]. A comparison with the results for Gadolinium and Uranium confirms the notion that the higher level densities present in the deformed rare-earth and actinide regions do reduce the sensitivity of the $\gamma$-decay probabilities to compound-nuclear spin-parity distributions and nuclear-structure effects [21].
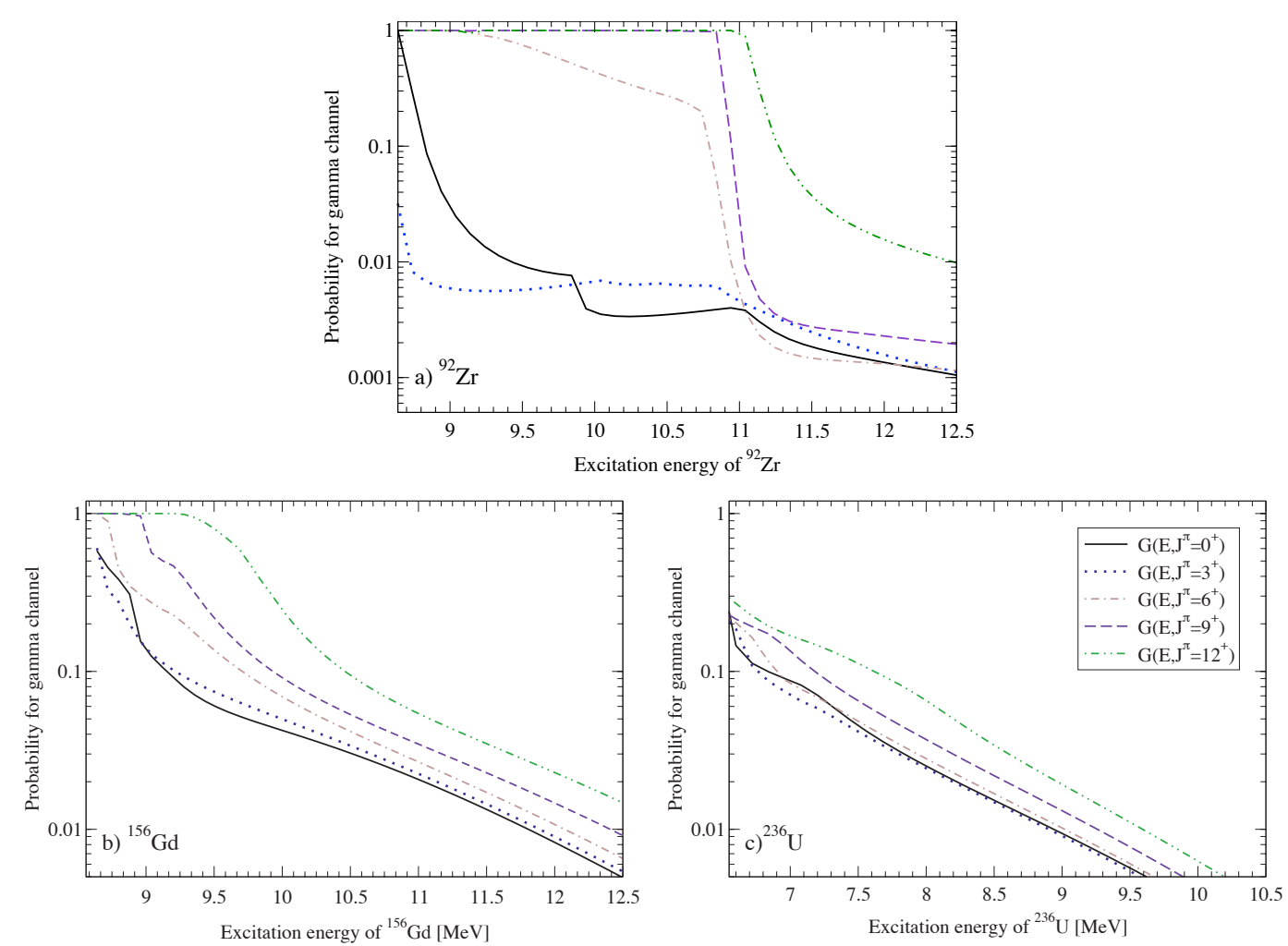

Fig. 3. Calculated $\gamma$-decay probabilities $G_{\gamma}^{C N}(E, J, \pi)$, for ${ }^{92} \mathrm{Zr},{ }^{156} \mathrm{Gd}$, and ${ }^{236} \mathrm{U}$. Shown is the probability that the compound nucleus, when produced with a specific $J \pi$ combination, decays via the $\gamma$ channel. The excitation energies shown correspond to incident-neutron energies of $0-4 \mathrm{MeV}$. The decay probabilities also depend on parity, only positive-parity results are shown here. More results can be found in [20,21].

The effects of the spin-parity mismatch can be further explored by using schematic spin-parity distributions, $F_{\delta}^{C N}(E, J, \pi)$, to simulate surrogate coincidence data via Eq. 2. The calculated $P_{\delta \gamma}^{\operatorname{sim}}(E)=$ $\sum_{J, \pi} F_{\delta}^{C N}(E, J, \pi) G_{\gamma}^{C N}(E, J, \pi)$ can be used in a WE 'analysis' to yield the desired cross section, $\sigma_{n, \gamma}^{W E, \operatorname{sim}}(E)=\sigma_{n}^{C N}(E) P_{\delta \gamma}^{\operatorname{sim}}(E)$, where $\sigma_{n}^{C N}(E)$ denotes the $\mathrm{CN}$ formation cross section. The range of cross sections, $\sigma_{n, \gamma}^{W E, s i m}(E)$, obtained by varying the simulated spin distributions within reasonable limits provides a measure of the uncertainty in the extracted cross section due to the use of the WE approximation. For the zirconium region such sensitivity analysis was carried out by Forssén et al. 


\section{CNR*11}

[20]. An order-of-magnitude difference between the known reference cross section for ${ }^{91} \operatorname{Zr}(\mathrm{n}, \gamma)$ and that extracted from the simulation was found, indicating that using the WE approximation for the this region of the nuclear chart is indeed not appropriate.
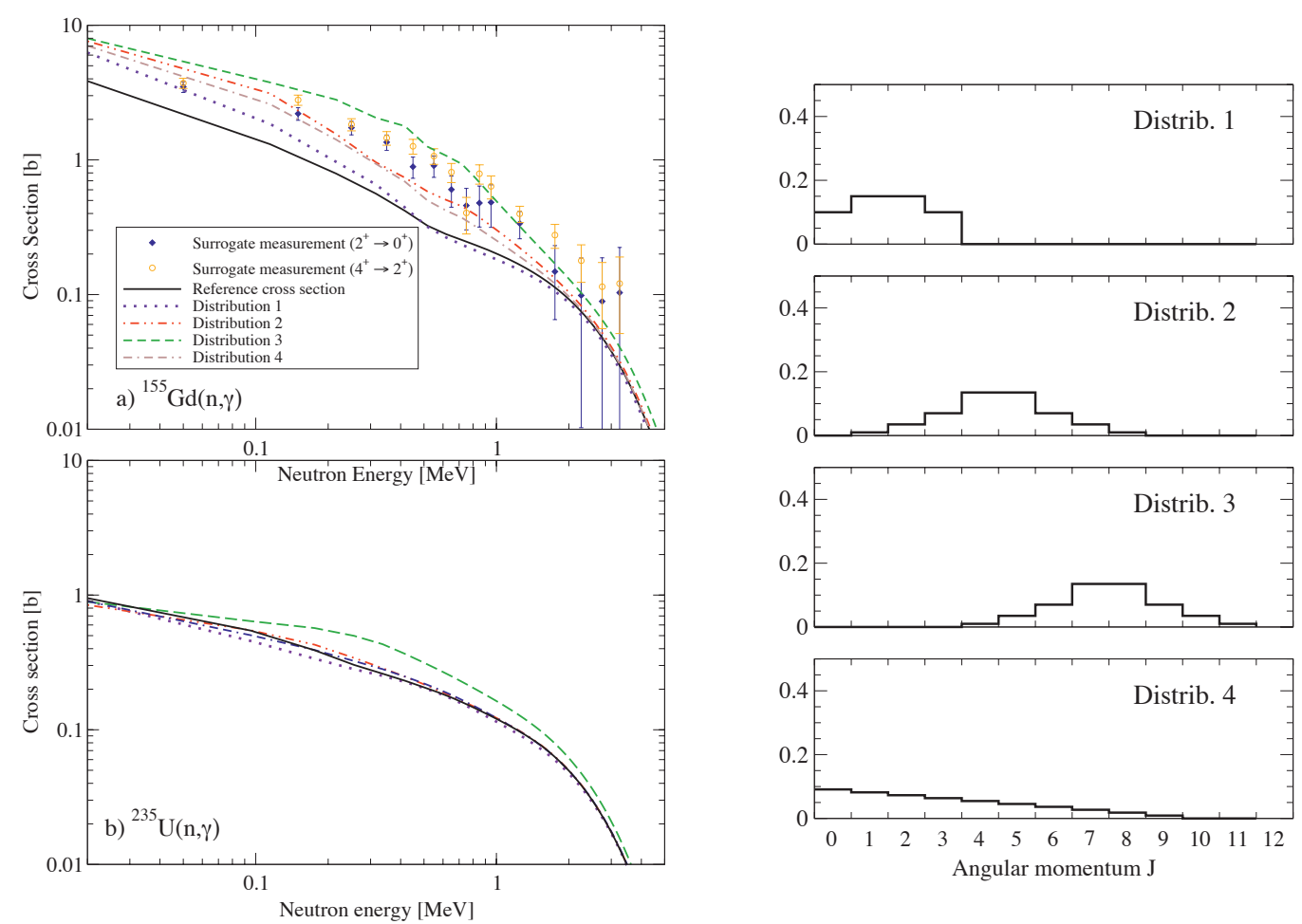

Fig. 4. Left panel: Weisskopf-Ewing estimates for the a) ${ }^{155} \mathrm{Gd}(\mathrm{n}, \gamma)$ and b) ${ }^{235} \mathrm{U}(\mathrm{n}, \gamma)$ cross sections, extracted from analyses of simulated surrogate experiments, for the four different compound-nuclear $J \pi$ distributions shown in the right panel of the figure. For the gadolinium case, results from a Weisskopf-Ewing analysis of measured surrogate ${ }^{156} \mathrm{Gd}(\mathrm{p}, \mathrm{p}$ ') data from Ref. [22] are also shown. The reference cross sections were obtained by adjusting the parameters for the Hauser-Feshbach calculation to reproduce direct $(\mathrm{n}, \gamma)$ measurements. Right panel: Schematic spin-parity distributions, selected to simulate the compound nucleus created in the surrogate reaction. Positive and negative parity states are assumed to be populated with equal probability.

Discrepancies between extracted and reference (i.e. evaluated) cross sections are expected to be smaller for the deformed rare-earth and actinide cases, since the level densities in those regions are much higher than in the zirconium region. We find that this is indeed the case. Results for the ${ }^{155} \mathrm{Gd}(\mathrm{n}, \gamma)$ and ${ }^{235} \mathrm{U}(\mathrm{n}, \gamma)$ examples are shown in Fig. 4 (see also Ref. [21]). Plotted are the reference cross sections, obtained by fitting Hauser-Feshbach calculations to direct measurements, and four cross sections extracted from simulated surrogate data; the spin distributions are shown in the right panel of the figure. For Gadolinium, we also included results from an actual surrogate experiment, carried out by the STARS/ LiBERACE collaboration at the 88-inch cyclotron at Lawrence Berkeley National Laboratory [22]. The symbols with y error bars indicate the cross section obtained from a WE analysis of a ${ }^{156} \mathrm{Gd}\left(p, p^{\prime}\right)$ measurement with $22-\mathrm{MeV}$ protons. Protons were detected in coincidence with the $\gamma$-ray from the $2^{+} \rightarrow 0^{+}$transition (blue diamonds), as well as the $4^{+} \rightarrow 2^{+}$transition (yellow open circles), in the ${ }^{156} \mathrm{Gd}$ ground-state band (no modeling was employed to account for $\gamma$-cascades that bypass these transitions). The extracted cross section falls, for the most part, between the calculated curves. It is somewhat larger than the curve corresponding to distribution 2 , which indicates that the $\left(p, p^{\prime}\right)$ reaction produced a spin-parity distribution which contained $J$-values above 5-6. The cross sec- 


\section{EPJ Web of Conferences}

tion extracted from the surrogate measurement is a factor of 2-3 larger than the reference cross section. The range of cross sections, $\sigma_{n, \gamma}^{W E, s i m}(E)$, obtained by varying the simulated spin distributions within reasonable limits provides a measure of the uncertainty in the extracted cross section due to the use of the WE approximation. Clearly, it is important to correct for the spin-parity mismatch.

For Uranium, we find that a Weisskopf-Ewing analysis of the simulated surrogate data leads to cross sections that are fairly similar to the reference cross section, see Fig. $4 \mathrm{~b}$. With the exception of distribution 3 , the curves are seen to cluster around the $(n, \gamma)$ reference result.

Surrogate Ratio approach. The studies of the Gadolinium region also show that the WeisskopfEwing approximation overestimates the $(\mathrm{n}, \gamma)$ cross section by factors which depend on the nucleus under consideration [21,22]. For the ${ }^{155} \mathrm{Gd}(\mathrm{n}, \gamma)$ and ${ }^{157} \mathrm{Gd}(\mathrm{n}, \gamma)$ cross sections, the differences are large, despite the structural similarities of the relevant nuclei. Consequently, the ratio approach results in ${ }^{155} \mathrm{Gd}(\mathrm{n}, \gamma)$ cross sections that are too large by up to a factor two for energies below about $E_{n}=$ $0.7 \mathrm{MeV}$ (Fig. 5a). The effect is seen in both the theoretical sensitivity study and the experimental results. Overall, the ratio approach was found to reduce, but not eliminate, the effect of the spin-parity mismatch on the extracted cross sections for energies where the Weisskopf-Ewing assumption is a poor approximation.
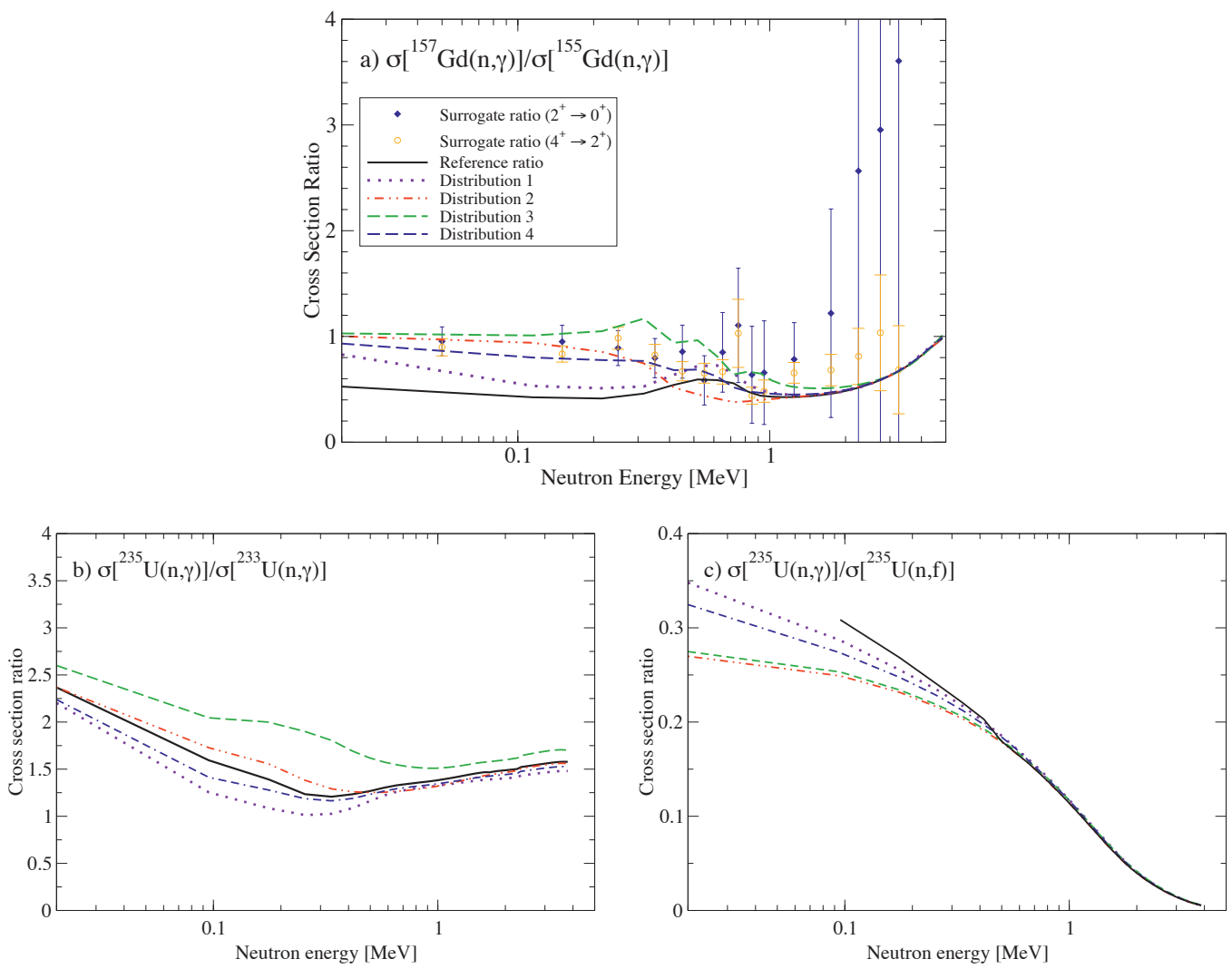

Fig. 5. (Color online) Cross section ratios obtained from surrogate data are compared to ratios of evaluated cross sections. The four different compound-nuclear $J \pi$ distributions shown in the right panel of Figure 4 were used to simulate surrogate data. In addition, experimental results from the surrogate measurement by Scielzo et al. [22] are plotted for gadolinium. a) External surrogate ratio approach for the ${ }^{157} \mathrm{Gd}(\mathrm{n}, \gamma)$ cross section; b) external surrogate ratio approach for the ${ }^{235} \mathrm{U}(\mathrm{n}, \gamma)$ cross section; c) internal surrogate ratio approach for the ${ }^{235} \mathrm{U}(\mathrm{n}, \gamma)$ cross section. 


\section{$\mathrm{CNR} * 11$}

Ratio results for Uranium are shown in Fig $5 \mathrm{~b}$ and c. For actinides, two types of ratio measurements can be considered: A distinction is made between internal and external surrogate ratio approaches, based on whether the ratio under consideration involves one compound nucleus (but different decay channels) or two different compound systems, but identical exit channels. The more widely employed variant is the external surrogate ratio method. In this variant, the cross sections in the ratio $R(E)=\sigma_{\alpha_{1} \chi_{1}}^{C N 1}$ $/ \sigma_{\alpha_{2} \chi_{2}}^{C N 2}$ of Eq. 4 refer to two reactions with the same type of entrance channel, $\alpha_{1}=\alpha_{2}(=n+$ target here $)$, and the same type of exit channel, $\chi_{1}=\chi_{2}(=\gamma$ decay here), but different compound nuclei, $C N 1 \neq C N 2$. An example is shown in Fig 5b, where we consider $\sigma\left[{ }^{235} \mathrm{U}(\mathrm{n}, \gamma)\right] / \sigma\left[{ }^{233} \mathrm{U}(\mathrm{n}, \gamma)\right]$. In the internal surrogate ratio approach, the compound nuclei created in the two reactions of interest are identical, $C N 1=C N 2$, the entrance channels are identical, $\alpha_{1}=\alpha_{2}$, but the decay channels differ in type, $\chi_{1} \neq \chi_{2}$. In Fig 5c, we show results for the decay of a compound uranium nucleus by $\gamma$ emission relative to the decay by fission. We find that the internal ratio approach shows closer agreement than the external surrogate ratio approach. Experimentally, the internal ratio method has been explored by Bernstein et al. [23] and by Allmond et al. [24].

\section{Gamma-ray cascades}

To improve the accuracy of the cross sections extracted from surrogate data and to reliably reliably apply it to neutron capture reactions, a more detailed description of the reaction mechanisms involved is required. Specifically, we have to quantitatively account for the fact that the weights $F_{\delta}^{C N}\left(E_{\mathrm{ex}}, J, \pi\right)$, by which the decay probabilities $G_{\chi}^{C N}\left(E_{e x}, J, \pi\right)$ are multiplied in Eq. 2, are different from the relative formation cross sections $f_{\alpha}^{C N}\left(E_{e x}, J, \pi\right) \equiv \sigma_{\alpha}^{C N}\left(E_{e x}, J, \pi\right) / \sum_{J^{\prime} \pi^{\prime}} \sigma_{\alpha}^{C N}\left(E_{e x}, J^{\prime}, \pi^{\prime}\right)$ of Eq. 1. Predicting these weights requires a framework for calculating cross sections of different reactions (stripping, pick-up, charge exchange, and inelastic scattering) to continuum states, for a variety of projectiles ( $p$, $d, t, \alpha$, etc.) and targets (spherical, deformed, and transitional).
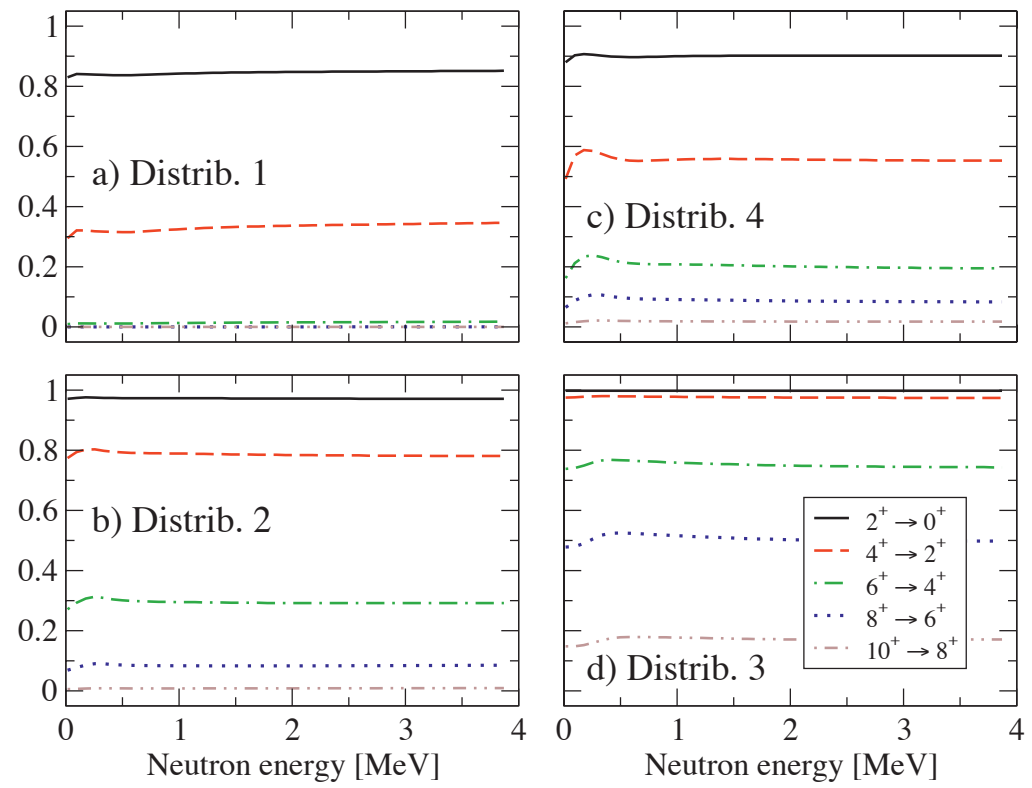

Fig. 6. Ratios of the yields of various $\gamma$-ray transitions in the ground-state band of ${ }^{236} \mathrm{U}$ to the total production of ${ }^{236} \mathrm{U}$, for the four schematic spin distributions shown in the right panel of Fig. 4. 


\section{EPJ Web of Conferences}

Theories describing transfer, inelastic scattering, and charge exchange reactions that lead to final states with low excitation energies are well established [25]. Many experiments have been carried out over the years to test the theoretical predictions against cross section measurements. The angular and energy dependences of the cross sections have been measured, for various projectile-target combinations and bombarding energies, and used to improve the theories. To use these theories to describe surrogate reactions they must be extended to treat highly-excited, overlapping, unbound final states in the residual nucleus. It is highly desirable that these extended theories be tested by measuring absolute cross sections for the energy and angular distributions of the ejectile, even though this is not strictly necessary to carry out a surrogate measurement.

For applications of interest here, it is not sufficient to calculate only the angular and energy dependence of the differential cross sections of the outgoing particle; reliable predictions for the spin-parity population of the residual compound nucleus are needed. Consequently, to test surrogate reaction models it is necessary to also identify observables that are sensitive to the $J \pi$ distribution of the compound nuclei created. Candidates include the yields of discrete $\gamma$-ray transitions of the decaying compound nucleus, as well as angular distributions of fission fragments, for systems that decay via this mode.

The path of the $\gamma$-cascade of a decaying compound nucleus is known to depend on the initial spin of the nucleus. While this fact can complicate the detection of the decay channel of interest in surrogate applications, it is also a source of useful information. Simultaneously measuring the yields of several such $\gamma$-ray transitions can provide signatures for the spin-parity distribution of the compound nucleus prior to decay. An example for this is shown in Fig. 6, where we have plotted the relative yields of several ground-state band transitions for ${ }^{236} \mathrm{U}$, for the four schematic $J \pi$ distributions shown in the right panel of Fig. 4. We find that different $J \pi$ distributions lead to markedly different relative $\gamma$-ray yields. These observables can be employed to test and constrain theories that predict compoundnuclear spin-parity distributions. Relative $\gamma$-ray yields for the decay of even-even gadolinium nuclei have recently been measured [22] and methods are being developed to use this information in order to improve the $(\mathrm{n}, \gamma)$ cross sections determined from surrogate experiments.

\section{Concluding Remarks}

Indirect approaches have to be developed in order to provide much-needed nuclear data, in particular cross sections for reactions on unstable isotopes. We have discussed the surrogate nuclear reaction method, which aims at providing cross section information for compound nuclear reactions. Past applications of the method have demonstrated that it can provide useful cross section estimates for neutron-induced fission of actinides. Similar success in applications to neutron capture for a range of isotopes would be very valuable, as capture cross sections play an important role in nuclear astrophysics, national security, and nuclear energy. Most analyses of fission data carried out so far have made approximations that are likely to break down in situations relevant for extracting $(\mathrm{n}, \gamma)$ cross sections from surrogate measurements, making this a more challenging reaction to tackle. The examples discussed illustrate this breakdown for capture reactions on Gadolinium isotopes and indicate that a similar breakdown can be expected for lighter nuclei near shell closures, while the situation for actinides seems slightly better. We have also indicated how the method can be improved by taking into account the different spin-parity distributions that occur in the desired and surrogate reactions. In particular, we have identified the role of gamma-ray cascades in testing theoretically predicted spin-parity distributions.

\section{Acknowledgments}

We thank R. Casperson for his help with Figure 1. This work was performed under the auspices of the U.S. Department of Energy by Lawrence Livermore National Laboratory under contract DE-AC5207NA27344, and supported in part by the Department of Energy's NNSA, Office of Nonproliferation Research and Development (NA-22). 


\section{References}

1. M.L. Cognata, C. Spitaleri, A.M. Mukhamedzhanov, The Astrophysical Journal 723(2), 1512 (2010)

2. R. Capote, M. Herman, P. Oblozinský, P. Young, S. Goriely, T. Belgya, A. Ignatyuk, A. Koning, S. Hilaire, V. Plujko et al., Nuclear Data Sheets 110(12), 3107 (2009)

3. E. Gadioli, P.E. Hodgson, Pre-Equilibrium Nuclear Reactions (Clarendon Press, Oxford, 1992)

4. J.E. Escher, J.T. Burke, F.S. Dietrich, N.D. Scielzo, I.J. Thompson, W. Younes, Rev. Mod. Phys. in press (2011)

5. W. Younes, H.C. Britt, Phys. Rev. C 67(2), 024610 (2003)

6. W. Younes, H.C. Britt, Phys. Rev. C 68(3), 034610 (2003)

7. J.D. Cramer, H.C. Britt, Nucl. Sci. and Eng. 41, 177 (1970)

8. H.C. Britt, J.B. Wilhelmy, Nucl. Sci. and Eng. 72, 222 (1979)

9. M. Petit, M. Aiche, G. Barreau et al., Nucl. Phys. A 735, 347 (2004)

10. S. Boyer, D. Dassie, J. Wilson, M. Aiche, G. Barreau, S. Czajkowski, C. Grosjean, A. Guiral, B. Haas, B. Osmanov et al., Nucl. Phys. A 775, 175 (2006)

11. G. Kessedjian, B. Jurado, M. Aiche, G. Barreau, A. Bidaud, S. Czajkowski, D. Dassié, B. Haas, L. Mathieu, L. Audouin et al., Physics Letters B 692(5), 297 (2010)

12. M. Basunia, R. Clark, B. Goldblum, L. Bernstein, L. Phair, J. Burke, C. Beausang, D. Bleuel, B. Darakchieva, F. Dietrich et al., Nuclear Instruments and Methods in Physics Research Section B: Beam Interactions with Materials and Atoms 267(11), 1899 (2009)

13. J.E. Escher, F.S. Dietrich, Phys. Rev. C 74(5), 054601 (2006)

14. C. Plettner et al., Phys. Rev. C 71, 051602(R) (2005)

15. J.T. Burke, L.A. Bernstein, J. Escher, L. Ahle, J.A. Church, F.S. Dietrich, K. Moody, E.B. Norman, L. Phair, P. Fallon et al., Phys. Rev. C 73, 054604 (2006)

16. S.R. Lesher, J.T. Burke, L.A. Bernstein, H. Ai, C.W. Beausang, D.L. Bleuel, R.M. Clark, F.S. Dietrich, J.E. Escher, P. Fallon et al., Physical Review C (Nuclear Physics) 79(4), 044609 (2009)

17. J.J. Ressler, J.T. Burke, J.E. Escher, C.T. Angell, M.S. Basunia, C.W. Beausang, L.A. Bernstein, D.L. Bleuel, R.J. Casperson, B.L. Goldblum et al., Phys. Rev. C 83(5), 054610 (2011)

18. F. Käppeler, A. Mengoni, Nuclear Physics A 777, 291 (2006)

19. G. Aliberti, G. Palmiotti, M. Salvatores, T. Kim, T. Taiwo, M. Anitescu, I. Kodeli, E. Sartori, J. Bosq, J. Tommasi, Annals of Nuclear Energy 33(8), 700 (2006), ISSN 0306-4549

20. C. Forssén, F. Dietrich, J. Escher, R. Hoffman, K. Kelley, Phys. Rev. C 75, 055807 (2007)

21. J.E. Escher, F.S. Dietrich, Phys. Rev. C 81(2), 024612 (2010)

22. N.D. Scielzo, J.E. Escher, J.M. Allmond, M.S. Basunia, C.W. Beausang, L.A. Bernstein, D.L. Bleuel, J.T. Burke, R.M. Clark, F.S. Dietrich et al., Phys. Rev. C 81(3), 034608 (2010)

23. L.A. Bernstein, J. Burke, L. Ahle, J.A. Church, J. Escher et al., Tech. rep., Lawrence Livermore National Laboratory, Livermore, CA (2006)

24. J.M. Allmond, L.A. Bernstein, C.W. Beausang, L. Phair, D.L. Bleuel, J.T. Burke, J.E. Escher, K.E. Evans, B.L. Goldblum, R. Hatarik et al., Physical Review C (Nuclear Physics) 79(5), 054610 (2009)

25. G.R. Satchler, Direct nuclear reactions (Oxford University Press, 1983) 